\title{
Effects of Core Dimensions and Manufacturing Procedure on Fluxgate Noise
}

\author{
M. JAnošEK ${ }^{a, *}$, J. VyhnáneK ${ }^{a}$, A. Zikmund ${ }^{a}$, P. Butvin ${ }^{b}$, B. Butvinová ${ }^{b}$
}

${ }^{a}$ Dept. of Measurement, FEE, Czech Technical University in Prague, Technická 2, 16627 Praha, Czech Republic

${ }^{b}$ Institute of Physics, Slovak Academy of Sciences, Dúbravská cesta 9, 84511 Bratislava, Slovakia

The effect of demagnetizing factor and manufacturing procedure on noise of the fluxgate probes, manufactured from metallic glasses, is presented. The fluxgate probes were either tape-wound, or flat, wet-etched ringcore and race-track geometries. When combining low demagnetizing factor and high enough cross-section of the probe, a minimum in the noise dependence can be found. For $50 \mathrm{~mm}$ ringcores, in such minimum the noise value as low as $7 \mathrm{pT}_{R M S}$ in 0.1-10 Hz frequency band was achieved, which is very low for an as-cast tape, and has not been reported before.

DOI: $10.12693 /$ APhysPolA.126.104

PACS: 75.75.-c, 75.30.-m, 75.50.-y, 07.55.-w, 75.70.-i

\section{Introduction}

The coupling of internal noise of the magnetic material to the noise of fluxgate sensors, manufactured from that material is ruled according to the commonly agreed practice, by the so called demagnetizing factor of the probe. This factor can be altered by changing the geometry of the probe (Fig. 1). For tape-wound sensors, core radius can be modified or the number of tape-turns can be altered. For ring-core and race-track geometries etched from tapes, changing the width of the annulus or stacking of the cores brings similar results.

The effect of the demagnetizing factor on noise was shown by Primdahl [1] for a given ringcore diameter of $17 \mathrm{~mm}$. Tape-wound racetracks, studied in [2], were also optimized by altering the race-track geometry.
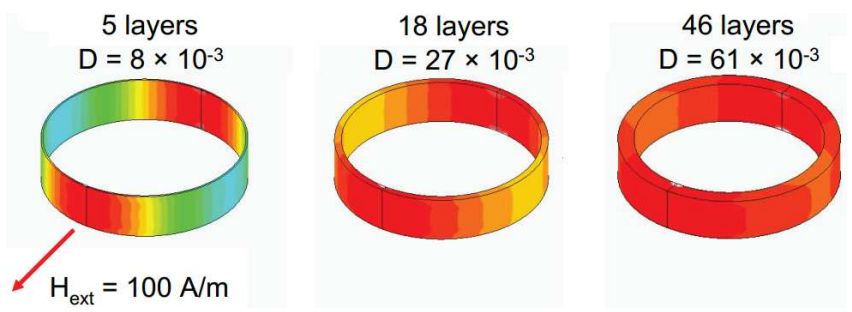

Fig. 1. Demagnetizing factor as a function of ringcore thickness (3D FEM simulation, the outer diameter $(12 \mathrm{~mm})$ is fixed).

However, mainly for ringcores with various diameters, the comparison is not straightforward: the bending stress, which becomes higher with smaller core diameter, is worsening the fluxgate probe noise. This can be handled either by appropriate zero-magnetostriction annealing [3], or at least by partially releasing the stresses

*corresponding author; e-mail: janosem@fel.cvut.cz during field annealing of the tape-wound core in its final form [4].

A different situation exists for flat cores wet-etched from a wide amorphous tape. In this case the bending stress does not affect the probe noise, however the smoothness of etching process and mainly the art of fixing the core to the bobbin influence the noise. Fluxgate probes with etched race-track cores were previously studied in printed-circuit-board (PCB) sensors; however the internal stresses due to embedding the core in the PCB laminate affected probe performance [5]. In [4] the authors have shown noise figure also for the race-track core which was not embedded in the laminate, however its performance was limited by excitation electronics. In this study, the working conditions were all the same for all studied sensors in order to facilitate the comparison of results.

\section{Material and geometry selection}

In this work, Vitrokov 8116 - a Co-Cr-Fe-B-Si metallic glass tape was used, with an average thickness of $20 \mu \mathrm{m}$ and with tape widths of $2.6 \mathrm{~mm}$ (wound cores) and $20 \mathrm{~mm}$ (etched race-tracks).

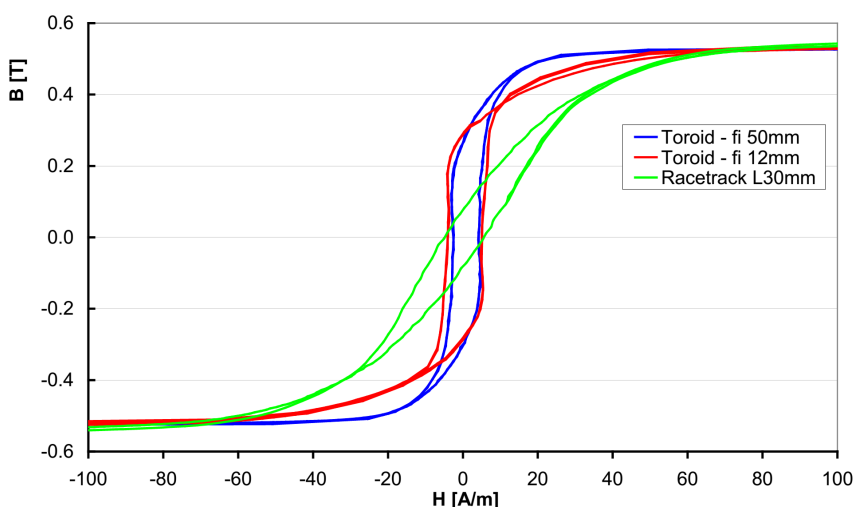

Fig. 2. $B-H$ loops of the 3 core geometries. 
For the tape-wound cores, the magnetoelastic effects can be seen by using the same material for $12 \mathrm{~mm}$ and $50 \mathrm{~mm}$ diameter (Fig. 2). From the $B-H$ loops it is evident, that even for these tapes with magnetostriction of $\sim 1 \times 10^{-7}$ this effect is present.

As for the $2.6 \mathrm{~mm}$ tape width, the material did not show any appreciable macroscopic heterogeneity, which otherwise causes poorly reproducible inhomogeneous magnetic anisotropy, brought about by macroscopic stress between surfaces and interior of many Febased ribbons (e.g. Finemets). The absence of this heterogeneity in Co-Cr-Fe-B-Si has been verified by comparing hysteresis loops prior to and after surfaces removal when the ribbon thickness was reduced by $10 \div 15 \%$ $(2 \div 3 \mu \mathrm{m})$.

In the $20 \mathrm{~mm}$ tape however, the heterogeneity was present but still acceptable. The different $B-H$ loop shape of the race-track in the comparison is evident but not caused by the tape macroscopic heterogeneity: the explanation lies in the as-cast anisotropy of the amorphous tape due to the manufacturing process, which is in the case of longitudinally etched race-tracks combined in both of the perpendicular branches of the race-track. An evidence for this behavior was shown in the MOKE pictures in [4].

\section{Noise measurements - setup and results}

The setup of electronics and operating conditions of the fluxgate probe were almost identical to that used in [4]. The resulting noise spectra for various tape thicknesses in the case of $50 \mathrm{~mm}$ ringcores obtained in a 6-layer magnetic shielding can, are shown in Fig. 3. To decrease the statistical error, an integral value of the noise in the frequency band of 0.1 to $10 \mathrm{~Hz}$ was calculated.

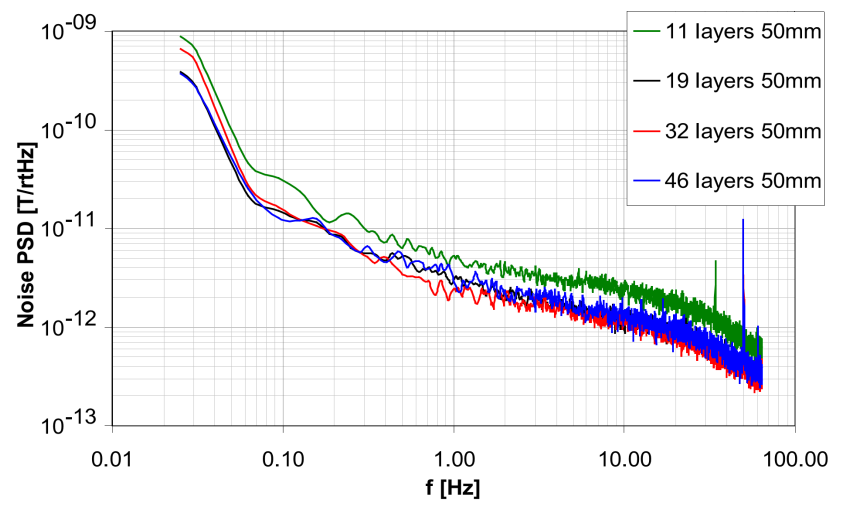

Fig. 3. Noise spectra of the $50 \mathrm{~mm}$ ringcore sensors.

The spectrum clearly maintains $1 / f$ behaviour with lowpass filtering visible for frequencies above $12 \mathrm{~Hz}$. The measuring procedure was done for the $50 \mathrm{~mm}$ cores in two tracks: 4 cores with different number of turns were produced and also one core was sequentially re-wound with decreased number of turns of the tape. The almost identical $50 \mathrm{~mm}$ results shown in Fig. 4 are thus independently obtained.

For $12 \mathrm{~mm}$ cores, there was a manufacturing problem of the bobbins, so only values for two demagnetizing fac- tors are shown ( 5 and 10 turns). Although no dependence can be deducted, the overall increased noise, due to the bending stress is evident. This was further confirmed by the one-layer, $30 \mathrm{~mm}$ long race-track noise: absence of the bending stress and relatively low demagnetizing factor resulted in a noise comparable to that of $50 \mathrm{~mm}$ wound cores.

The results are in agreement with the behaviour found by Primdahl [1]. The noise for a given geometry slowly decreases with the decreasing demagnetizing factor up to a given minimum, from which it increases much faster.

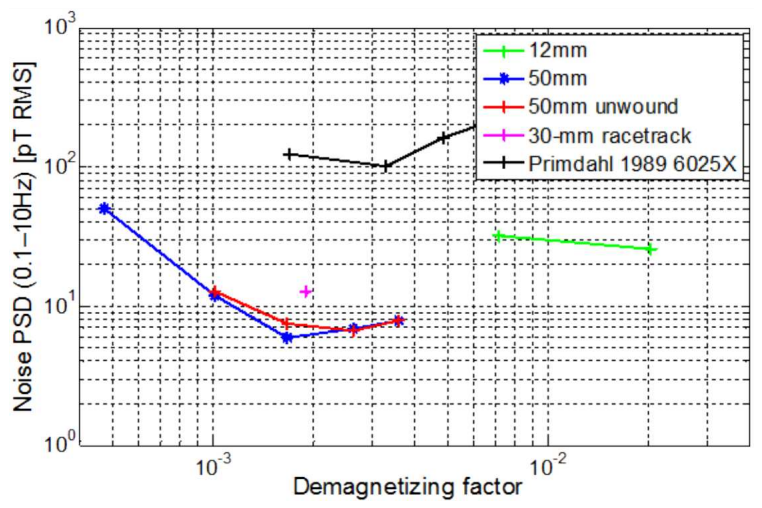

Fig. 4. Noise vs. demagnetizing factor for three geometries, including results of Primdahl for reference (he used 5, 10, 15 and 20 turns, respectively, on $17 \mathrm{~mm}$ diameter).

The authors offer the following explanation: as the cross-section of the magnetic material decreases in this case, also the signal-to-noise ratio of the whole sensorelectronics setup decreases, so the lower demagnetizing factor cannot bring a benefit anymore. Here the limitation was not the $5 \mathrm{nV}$ lock-in amplifier noise, but rather remanent parasitic signal from unwanted even harmonics in the excitation current, which does not change when the core cross-section (and thus demagnetizing factor) is altered.

\section{Acknowledgments}

The authors are grateful for partial financial support by APVV grant SK-CZ-0078-11.

\section{References}

[1] F. Primdahl, B.Hernando, O.V. Nielsen, J.R. Petersen, J. Phys. E: Sci. Instrum. 22, 1004 (1989).

[2] C. Hinnrichs, J. Stahl, K. Kuchenbrandt, M. Schilling, IEEE Trans. Mag. 37, 1983 (2001).

[3] F. Primdahl, P. Brauer, J.M.G. Merayo, O.V. Nielsen, Meas. Sci. Technol. 13, 1248 (2002).

[4] P. Butvin, M. Janošek, P. Ripka, B. Butvinová, P. Švec sr., M. Kuzminski, P. Švec jr., D. Janičkovič, G. Vlasák, Sens. Act. A 184, 72 (2012).

[5] J. Kubík, L. Pavel, P. Ripka, Sens. Act. A 130-131, 184 (2006). 